\title{
Using Non Local Features for 3D Shape Grouping
}

\author{
Antonio Adán ${ }^{1}$, Miguel Adán ${ }^{1}$, Santiago Salamanca ${ }^{2}$, and Pilar Merchán ${ }^{2}$ \\ ${ }^{1}$ Universidad de Castilla La Mancha. Escuela Superior de Informática. Ciudad Real. Spain \\ ${ }^{2}$ Universidad de Extremadura. Escuela de Ingenierías Industriales. Badajoz. Spain \\ antonio.adan@uclm.es, miguel.adan@uclm.es, ssalaman@unex.es, \\ pmerchan@unex.es
}

\begin{abstract}
This work faces the problem of 3D shape clustering when the whole surface information is available. The key of our method is to use a flexible feature, called Cone-Curvature, which provides local and extended information around every node of the mesh that represents the object. Thus as we increase the region around a node a new order of CC can be calculated. This feature, which was originally defined on spherical representation, has been adapted to work with standard triangular meshes and it is used for defining a similarity measure between shapes. Through a PCA technique, the dimensionality of the shape representation is drastically reduced and the hierarchical grouping can be efficiently carried out. This method has been tested under real conditions for a wide set of free shapes yielding promising results. We present a discussion of the clustering comparing human and computer results.
\end{abstract}

Keywords: Object clustering, Shape similarity, 3D representation, 3D models.

\section{Introduction}

3D shape clustering is an old topic on which many researchers continue working. From a human viewpoint the similarity is an ambiguous and relative concept. Intuitively, we put two objects in the same group taking into account the external appearance or some local/global features, but we can not be sure if we are right. Thus, different results depending on the perception of each person may be obtained [1]. Anyway, the similarity between two shapes is usually determined as a distance between some extracted features.

Roughly speaking, the clustering's goal is to achieve the best partition over a set of objects stored on a database in terms of similarity. For partitioning we need to extract or define features of each object in such a way as to be well characterized.

Similarity-base clustering is a simple technique that uses a similarity measure to guarantee if two objects are similar enough to put them in the same cluster. The similarity measure is usually defined through features of the object. In this sense, a good similarity measure is essential to carry out further clustering tasks.

As we know, there is a wide variety of approaches that propose different solutions to compare objects. In 3D environments, most of them use representation models based on geometrical descriptors and establish similarity relationships by means of feature matching procedures. 
Local features describe the geometry of little regions close to a set of nodes (or vertices) of the mesh that represent the object. Thus, every point of the surface of the object has knowledge of the features of its immediate neighbours. Geometrical features such as simplex angle [2], curvature index [3] and integral Gaussian curvature [4], [5] correspond to these kinds of features. For a mesh representation, this means that a given node is handled as a single item isolated from the complete mesh and without any relationship to the remaining nodes. Usually these techniques are addressed to solve recognition and pose problems in occlusion circumstances more than for clustering purposes. Some of the most known techniques are as follows. Dorai and Jain [6] use a local curvature feature called shape index and define a disimilarity measure by comparing their histograms for several moments. Johnson and Hebert [7] compare two objects through spin image concept. In [8,9] (Yamany and Farag) a representation that stores surface curvature information from certain points, produces images, called surface signatures, at these points. As a result of this, a standard Euclidean distance to match objects is presented. Chua and Jarvis code surroundings information at a point of the surface through a feature called Point Signature [10].

On the other hand, methods that consider global characteristics are more suitable for carrying out clustering solutions because, contrary to local strategies, they define the whole shape and are not correlated with the observer viewpoint. Vandeborre, Couillet and Daoudi [11] obtain three distances by comparing local and global feature histograms. Adán et. al. [12], propose a recognition process where several distances, based on invariant global features, sequentially reduce the original model database until the object is identified. Liu, Sun, Kang and Shum propose the Directional Histogram Model for objects that have been completely reconstructed [13]. For each viewpoint, they obtain the histogram of thickness distribution that is later formulated with spherical harmonics.

\section{A Flexible 3D Shape Descriptor}

Adaptable strategies are rarely considered in literature. In this case, every node of the model has an extended knowledge of its surroundings and all or part of it can be used for further tasks. The main difficulty in using that idea, in a solid modeling environment, is to define a model with an appropriate topology and connectivity.

The models MWS (Modeling Wave Set) [14] have been designed to maintain new and wider relationships among subsets of mesh nodes. Contrary to most strategies, MWS structures allow us to compare two objects taking any part of information from the model. So, local, half, global, contiguous or discontiguous spatial information could be chosen to recognize an object. In order to express this concept of adaptability to different specifications, we have labelled our method as 'flexible'. Consequently, an adaptable (or flexible) similarity measure contrary to previous fixed similarity measures is defined in our case.

MWS was originally defined on a mesh of nodes with a 3-connectivity relationship from the tessellation of the unit sphere. This representation, with a constant number of nodes, has been efficient in solving recognition problems in medium database but with some shape limitations. This is due to the fact that a poor realistic geometric representation is yielded with such a mesh. In this work, we have extended MWS 
structure to a semi-regular triangular mesh which involves three evident improvements with respect to the original version. Firstly, we can use the standard topology in most of the CAD tools and 3D scanner software. Secondly, we achieve a realistic representation where the appearance of the object is as accurate as possible. As a consequence of this, old shape restrictions are now avoided and $3 \mathrm{D}$ clustering can be carried out more efficiently.

Let $\mathrm{T}$ be a triangular mesh fitted to the object and let $\mathrm{N}$ be a vertex of $\mathrm{T}$. Note that $\mathrm{T}$ has been previously regularized and resampled to a fixed number of nodes $h$. Modeling Wave (MW) organizes the rest of the nodes of the mesh in concentric groups spatially disposed around N. Since this organization resembles the shape of a wave it is called Modeling Wave $(M W)$. Consequently, each of the disjointed subsets is known as Wave Front $(W F)$ and the initial node $\mathrm{N}$ is called Focus. Let us call all the possible MWs that can be generated over T Modeling Wave Set (MWS). Figure 1 illustrates the mesh model of an object and several wave fronts plotted for different focuses.
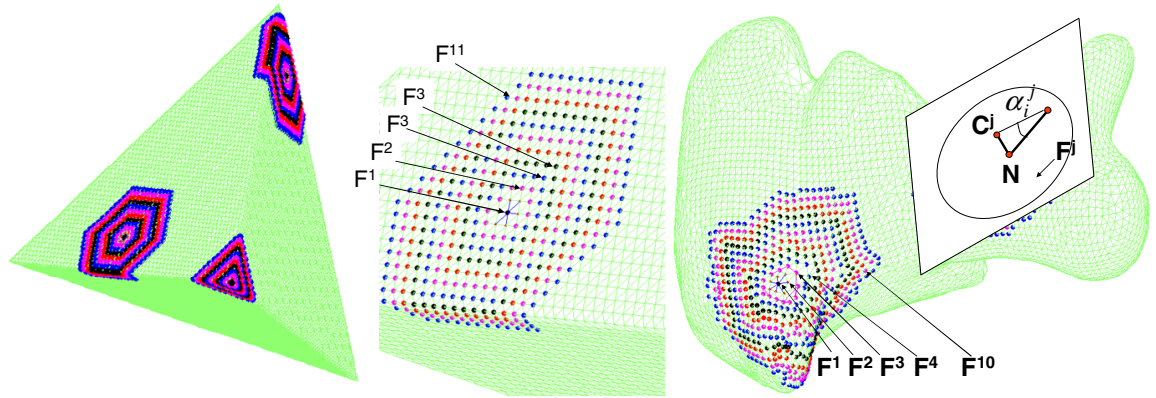

Fig. 1. Wave fronts in different focuses printed over the mesh model (left). Representation of jth cone curvature in a point of the object (right).

Cone Curvature is defined taking into account MWS structure. We define Cone Curvature $\alpha^{j}$ of $\mathrm{N} \in \mathrm{T}$, the angle of the cone with vertex $\mathrm{N}$ whose surface inscribes the jth Wave Front of the Modeling Wave associated to N.

The range of $\mathrm{CC}$ values is $[-\pi / 2, \pi / 2]$, being the sign assigned taking into account the relative location $C^{j}$ with respect to $\mathrm{T}, C^{j}$ being the barycentre of the $j$ th WF. Figure 1 right illustrates this definition and shows examples for positive and negative cases.

Note that a set of values $\left\{\alpha^{d}, \alpha^{2}, \alpha^{3}, \ldots \alpha^{q}\right\}$ gives an extended curvature information around $\mathrm{N}$ until the $q$ th $\mathrm{WF}$, where the word 'curvature' has a non-local meaning. So for each node $\mathrm{N}$ a set of $q$ values could be used for exploring its surroundings

On the other hand, it can be said that a vector

$$
V_{C C}=\left\{c^{1}, c^{2}, c^{3} \ldots, c^{q}\right\}, c^{j}: T \rightarrow[-\pi / 2, \pi / 2] \quad j=1, \ldots q
$$

$q$ being the number of Wave Front considered, is established for all the nodes of T. Then the whole Cone Curvature information is stored in a CC-matrix $M_{C C}$, of $h \times$ 
dimension. Note that $M_{C C}$ is invariant, unless row permutations, to changes in the pose of the object. A wider explanation of CC concept can be found in [15].

The main properties are briefly mentioned as follows. Figure 2 illustrates a visualization of those, where the $\mathrm{CC}$ value is plotted over the mesh in a specific color code.

- CC is robust to noise. Small variations in the location of the nodes in the mesh, involve high variations in first orders of CC whereas for upper orders such variations do not meaningfully affect it. It can be said that an error filtering effect appears as the order increases. Keeping this in mind, local features are not suitable enough for characterizing an object unless errors are minimized.

- Spatial continuity of CC. It means that CC along any path of nodes is a continuous function for any $\mathrm{CC}$-order considered. A visualization of this property, in the discrete sense, can be appreciated in Figure 2 where the $\mathrm{CC}$ value is plotted over the mesh in a specific code colour.

- Continuity on the CC-order. It can be said that there is also continuity in the CC vector $\left\{\alpha^{l}, \alpha^{2}, \ldots \alpha^{q}\right\}$. In other words, CC of every node of the mesh smoothly changes as the order grows.

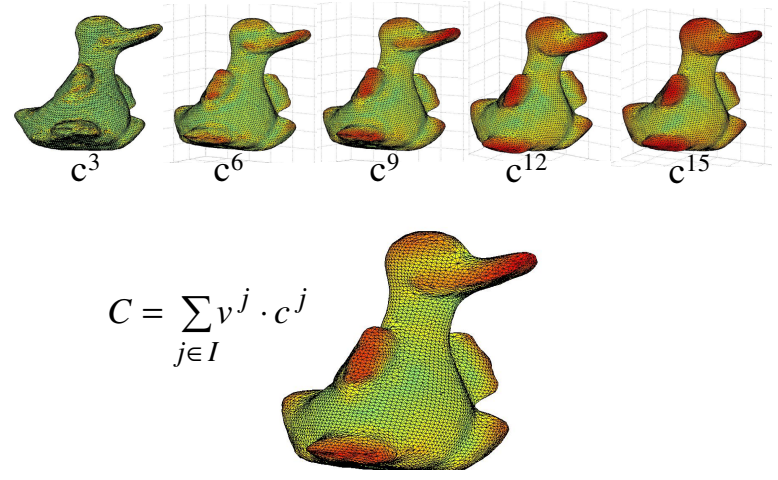

Fig. 2. Illustration of several CC orders (above) and the weighted cone curvature after considering from third until the 15-th CCs

\section{Hierarchical Grouping}

The hierarchical clustering algorithm is carried out after reducing the CC-matrix $M_{C C}$ to a single vector. Our purpose is to obtain a single value for each row of the matrix $M_{C C}$ from the analysis of the principal components performed on all the rows, so that each row is reduced to a single representative value. Then, by means of the adequate linear combination, for each node $\mathrm{N}$, a single variable $C$ will fuse the $q$ values provided by its CCs. Therefore, every node will have an associated variable $C$ that is called Weighted Cone Curvature (WCC). Consequently, the matrix $M_{C C}$ is reduced to a vector of $h x l$ dimension.

Formally, the variable Weighted Cone Curvature can be defined as a weighted combination of the different orders of CCs given by the expression: 


$$
C=\sum_{j \in I} v^{j} \cdot c^{j}
$$

where $j$ is extended to any set of CC-orders. Thus, we can choose contiguous or not contiguous wave fronts, near or far from the focus depending on the specifications of the experiment and the kind of shapes. Therefore, if we choose $j$-th CC we know exactly what it is because it is inside the whole structure MW. Therefore CC is not an isolated concept. This makes our representation more compact than others $([7,8,9,10]$.

Coefficients $v^{j}$ in expression (2) are the coordinates of the eigenvector associated to the highest eigenvalue of the covariance matrix for the set of indexes $I$ considered. These coefficients have been empirically determined by evaluating the principal component analysis over the models of our object database in our lab.

We define the distance $d$ between two 3D shapes through their models $T^{i}$ and $T^{j}$ as follows:

$$
d\left(T^{i}, T^{j}\right)=\left|C^{i}-C^{j}\right|=\sqrt{\sum_{k=1}^{h}\left(C^{i}(k)-C^{j}(k)\right)^{2}}
$$

where $C^{i}$ and $C^{j}$ are sorted values of WCC vectors for both models.

Once the distance $d$ has been defined and considering a model database a similarity function is established as follows.

$$
s\left(T^{i}, T^{j}\right)=s_{i j}=1-d\left(T^{i}, T^{j}\right) / \max \left\{d\left(T^{i}, T^{j}\right), \forall i, j\right\}
$$

Finally we can define a Similarity Matrix $S=\left(s_{i j}\right)$ which stores the whole similarity information for a database.

There are two major methods of clustering: hierarchical clustering and k-means clustering. We have applied the hierarchical clustering method based on the Similarity Matrix $S$. As it is known, in hierarchical strategies the data (in our case $S$ ) are not partitioned into a set of groups in a single step. Instead, a series of partitions takes place, which may run from a single cluster containing all objects to $m$ clusters each containing a single object. Hierarchical Clustering is subdivided into agglomerative methods, which proceed by series of fusions of the $m$ objects into groups, and divisive methods, which separate $m$ objects successively into finer groupings. The result may be represented by a dendogram which illustrates the fusions or divisions made at each successive stage of analysis.

Among the different ways of measuring the distance between two clusters of objects, we have chosen the Ward's linkage which uses the incremental sum of squares; that is, the increase in the total within-cluster sum of squares as a result of joining two clusters. The within-cluster sum of squares is defined as the sum of the squares of the distances between all objects in the cluster and the centroid of the cluster. For clusters $r$ and $s$ the equivalent distance is given by:

$$
d^{2}(r, s)=n_{r} n_{s} \frac{\left|\bar{x}_{r}-\bar{x}_{s}\right|^{2}}{n_{r}+n_{s}}
$$


where I.I is Euclidean distance, $\bar{x}_{r}$ and $\bar{x}_{s}$ are the centroids, $n_{r}$ and $n_{s}$ are the respective number of objects in cluster $r$ and $s$.

\section{3D Shape Clustering Experimental Test}

The hierarchical method proposed in this article has been verified using synthetic as well as real range data from a 3D laser scanner. We have built a database of 115 objects where 40 of them are models corresponding to real objects which have been built using a MINOLTA 910 scanner, 40 correspond to synthetic shapes which have been generated from CAD software and the rest were obtained deforming all those through smoothing, sharpening and bending processes. In this paper we present an experiment in which the clustering result is seemingly predictable for a human (see Figure 3).

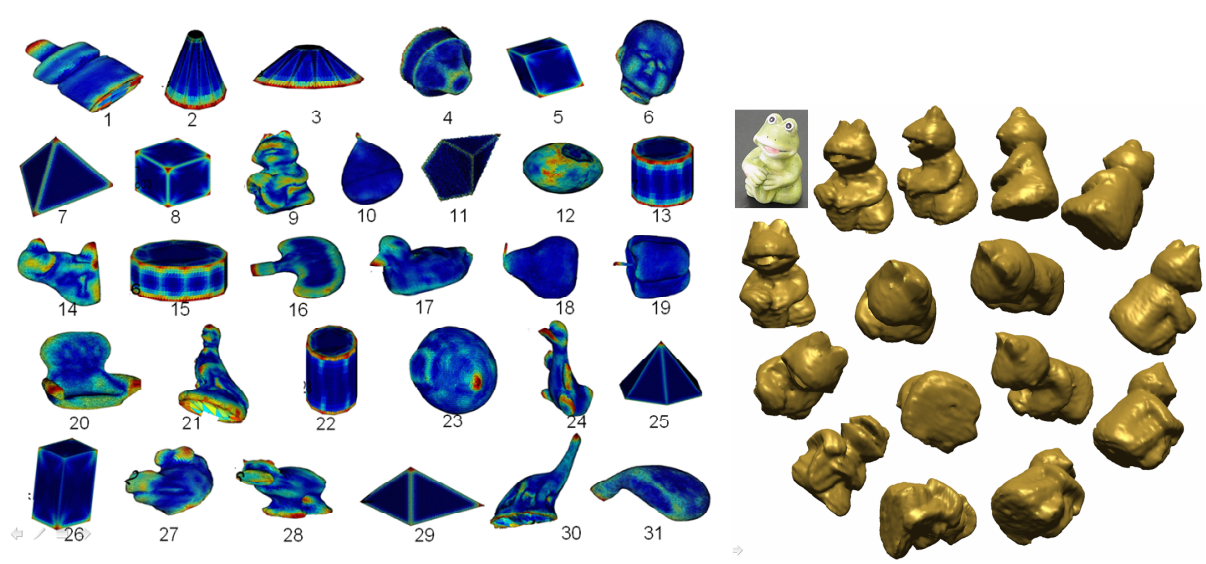

Fig. 3. Left: Objects database B. The colour code printed in the model mesh corresponds to the weighted cone curvature values. Right: synthetic model explored from multiple viewpoints.

Ten people were asked to perform the clustering procedure together over a selected database of 31 objects. A 3D visualization tool helped people to see the objects from any viewpoint (Figure 3 right). In order to compare the results of this experiment to those computed by our method, we asked them to carry out a splitting procedure in which the group of objects was iteratively separated into two parts until the end. So, after a consensus between all persons, a human dendogram $\Lambda^{\prime}$ was built. This is shown in figure 4 . No conditions were imposed upon the number, the names of the groups and the belonging of each object to a specific group. The only requirement was to apply shape criteria.

On the other hand, we obtained the hierarchical cluster tree $\Lambda$ using the minimum variance criteria within-clusters according to the Ward's linkage (see Figure 6). In this paper we only show the results obtained for weighted cone curvatures corresponding to orders 1 to 13 . 


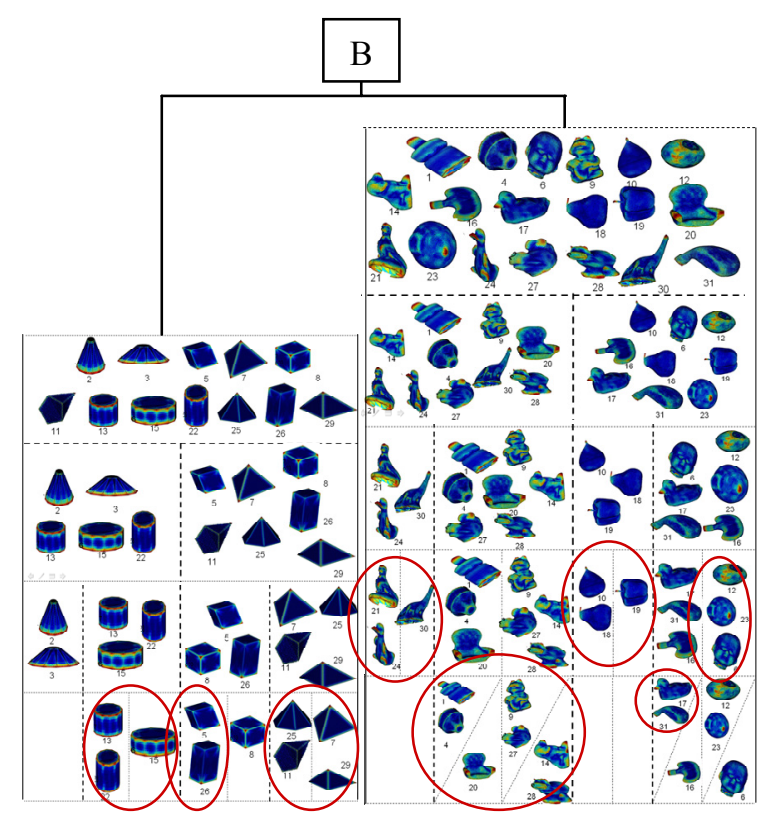

Fig. 4. Human dendogram $\Lambda^{\prime}$ built after consensus of ten persons. Coherent groups with the computed dendogram are marked on it.

Firstly, we present a qualitative evaluation of the results compared with human clustering. It can be seen that different clusters appear depending on the depth taken in the dendogram. Firstly, our algorithm gives a classification into two groups which we have labeled as polyhedral and free-form objects. This is an expected and reasonable division which agrees with the first division in $\Lambda^{\prime}$. Among polyhedral objects we discussed what groups could be similar in $\Lambda^{\prime}$ and what label should be assigned to them. Finally we found Pyramids (I), tetrahedrons (II), paralelepipeds (III) and cylinders (IV). Curiously the cube (object 8) is more similar to cylinders than to paralelepideps and also two cones are not put in the same groups in the last level. Concerning free-form group we distinguish five groups, some of them appear in the tree $\Lambda^{\prime}$. For instance we find round objects (X) and elongated objects (IX). Group VIII are also rounded but with a point at its end. Finally, groups VI and VII are difficult to understand and to label.

In order to provide a quantitative analysis of our approach, a concordance measure between groups of $\Lambda$ and $\Lambda^{\prime}$ has been defined using a vote strategy. Let $a$ and $a^{\prime}$ be two groups, $a \in \Lambda$ and $a^{\prime} \in \Lambda^{\prime}$. The concordance of group $a$ in the tree $\Lambda^{\prime}$ is defined as:

$$
\begin{gathered}
H\left(a, a^{\prime}\right)=e^{-\frac{\left|V\left(a, a^{\prime}\right)-n_{a}\right|}{n_{a}}} \\
V\left(a, a^{\prime}\right)=\max \left\{\sum_{o \in a} \delta\left(o, a^{\prime}\right)+\sum_{o \in a^{\prime}} \delta(o, a)\right\}
\end{gathered}
$$

where $n_{A}$ is the number of objects in $A$ and $\delta$ is the voting function 


$$
\delta\left(o, a^{\prime}\right)=\left\{\begin{array}{ll}
1 & o \in a^{\prime} \\
-1 & o \notin a^{\prime}
\end{array} \quad \delta(o, a)= \begin{cases}1 & o \in a \\
-1 & o \notin a\end{cases}\right.
$$

Obviously, $H$ is not a symmetrical matrix where the $i$-th column represents the concordance of the $i$-th group of $\Lambda$ in $\Lambda^{\prime} . H$ is a normalized function in the range [0,1], where $H\left(a, a^{\prime}\right)=1$ if $a \equiv a^{\prime}$ and $H\left(a, a^{\prime}\right) \rightarrow 0$ if any object of $a$ is in $a^{\prime}$.

In order to graphically illustrate $H$, we have presented it on a two dimension grey map where the grey level goes from the minimum value $(0)$, plotted in black, to the maximum (1), plotted in white (Figure 5 left). In this figure, we have ranked $\mathrm{H}$ with respect to the computed clustering $\Lambda$. Note that we consider all subgroups that are generated throughout the whole splitting process and that the number of cluster generated in both dendograms is different (40 in $\Lambda$ and 36 in $\Lambda^{\prime}$ ). Looking at this figure we can appreciate high concordance between dendograms $\Lambda$ and $\Lambda^{\prime}$ in several points. The clearer $H$, is the more concordance between $\Lambda$ and $\Lambda^{\prime}$.

Figure 5 right gives a more extended analysis taking into account the $H$ value of the groups in which an object is classified along the tree $\Lambda$. In this figure we represent, for each one of the objects of the database, the average value of those values and the standard deviation. Finally, respective global mean values are calculated and pointed out in this figure superimposing a horizontal line. These measures provide a qualitative idea of whether an object is classified in more or less concordant groups throughout the whole clustering process. This means that, in a general sense, some objects are expected to be better classified than others. For instance, objects 11, 22 and 25 have average values above 0.8 whereas objects 2,4 and 6 have values below 0.6 . The global mean value was 0.72 .

In figures 4 and 6 we have marked the groups with a high concordance measure. Groups with $H$ values between 0.5 to 1 are pointed out by means of a coloured spot plotted on the division points. Note that the results in this experiment are surprising.

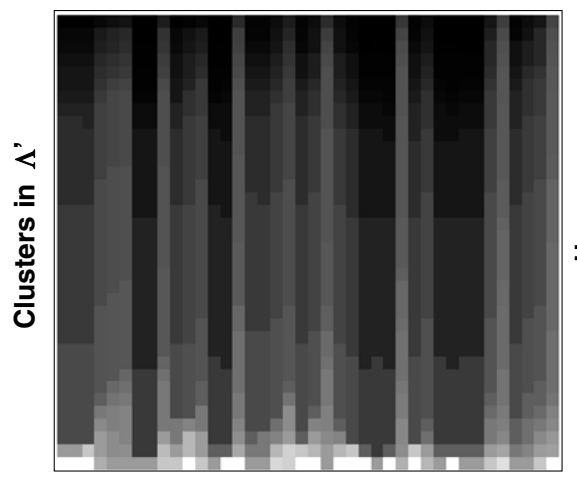

Clusters in $\Lambda$

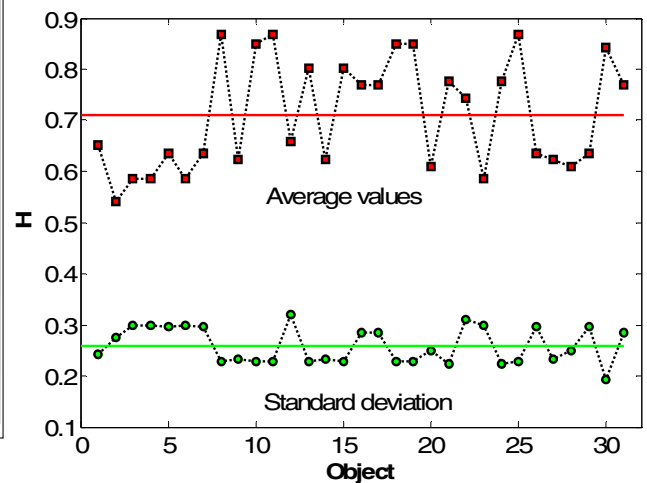

Fig. 5. Left: Comparison of clusters $\Lambda$ and $\Lambda^{\prime}$ through $H$. Ordered values of $H$ for each one of the groups generated in $\Lambda$. Right: Average values of $\mathrm{H}$ per object. 


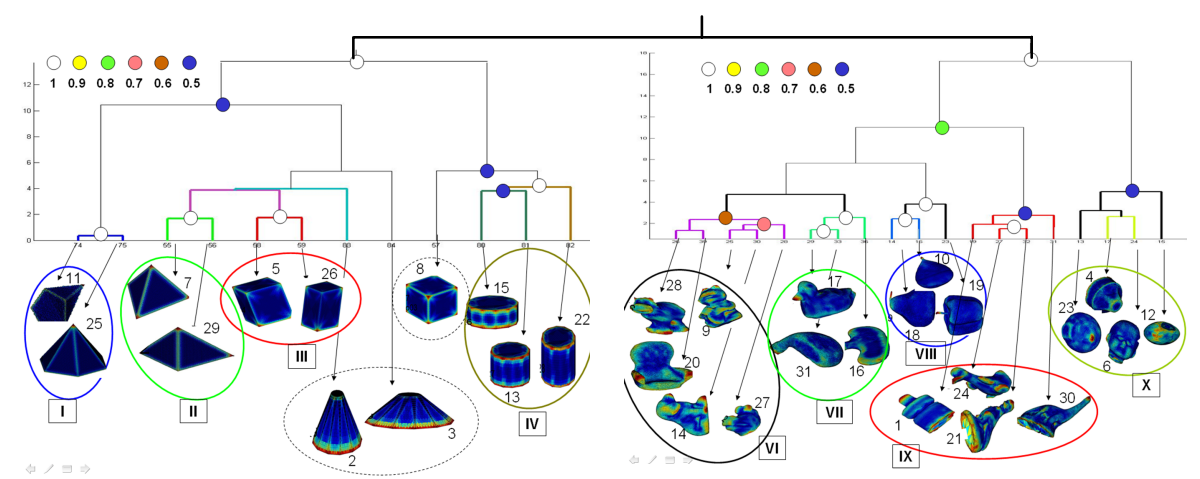

Fig. 6. Computed dendogram $\Lambda$. The groups with high concordance measure $H$ are pointed out by means of coloured spots. Interesting groups in the last levels, with a notable value $\mathrm{H}$, are also marked.

Thus $84 \%$ of the objects are classified in coherent groups with $H$ values higher than 0.5 , whereas only $16 \%$ (objects $2,3,8,12$ and 30 ) is put in unexpected groups or appear as isolated objects.

In conclusion we can say that clustering based on cone-curvature features can yield very good results.

\section{Conclusions}

Clustering is a well known topic in the image processing field. Roughly speaking, the clustering's goal is to achieve the best partition over a set of objects stored in a database in terms of similarity. This may become a tricky problem in $3 \mathrm{D}$ environments particularly when only shape information is considered.

For partitioning 3D shape databases we have used a flexible and adaptable feature which totally characterizes each object. This feature, called Cone Curvature, and originally defined on spherical representation models, has been adapted to standard triangular meshes and further used for solving clustering problems.

It can be said that the hierarchical clustering method has worked in a real environment which implies working with noise and error sources. In general, the experiments have yielded coherent and expected clustering results. Thus, high concordance between computed and human dendograms has been found. Nevertheless, we have started several initiatives to improve the method in two ways: firstly, to cope with a higher database and more shapes and, secondly, to cope with more exhaustive clustering evaluation.

\section{Acknowledgment}

This work is supported in the following projects: DPI2006-14794-C02 and PCI080052-1401 by the National and Regional Ministries of Education and Science. 


\section{References}

[1] Santini, S., Jain, R.: Similarity measures. IEEE Trans. Pattern Anal. Mach. Intell 21(9), 871-883 (1999)

[2] Delinguette, H.: Simplex Meshes: A General Representation for 3D Shape Reconstruction, Technical Report 2214, INRIA, France (1994)

[3] Koenderink, J.J., Van Doorn, A.J.: Surface shape and curvature scales. Image and Vision Computing 10(8), 557-564 (1992)

[4] Dyn, N., Hormann, K., Kim, S.J., Levin, D.: Optimizing 3D Triangulations Using Discrete Curvature Analysis. In: Mathematical Methods for Curves and Surfaces, pp. 135146. Vanderbilt University Press (2000)

[5] Alboul, L., Van Damme, R.: Polyhedral metrics in surface reconstruction. In: Mullineux, G. (ed.) The Mathematics of Surfaces VI, pp. 171-200. Clarendon Press, Oxford (1996)

[6] Dorai, C., Jain, A.K.: COSMOS - A Representation Scheme for 3D Free-Form Objects. IEEE Trans. PAMI 19(10), 1115-1130 (1997)

[7] Johnson, A.E., Hebert, M.: Recognizing Objects by Matching Oriented Points. In: IEEE Conf. Comp. Vision and Pattern Recogn, Puerto Rico, June, pp. 684-689 (1997)

[8] Yamany, S.M., Farag, A.A.: Free-Form Surface Registration Using Surface Signatures. In: Proc. IEEE Intern. Conf. on Computer Vision, vol. 2, pp. 1098-1104 (1999)

[9] Yamany, S.M., Farag, A.A.: Surfacing Signatures, An Orientation Independent FreeForm Surface Representation Scheme for the Purpose of Objects Registration and Matching. IEEE Trans. Pattern Anal. Mach. Intell 24(8), 1105-1120 (2002)

[10] Chua, C.S., Jarvis, R.: Point Signatures, A New Representation for 3D Object Recognition. International Journal of Computer Vision 25(1), 63-85 (1997)

[11] Vandeborre, J.-P., Couillet, V., Daoudi, M.: A practical approach for 3D model indexing by combining local and global invariants. In: $1^{\text {st }}$ Int. Symp. On 3D Data Processing Visualization and Transmission, Padova, Italy, pp. 644-647 (2002)

[12] Adán, A., Cerrada, C., Feliu, V.: Global Shape Invariants: A Solution For 3D Object Discrimination/ Identification Problem. Pattern Recog. 34, 1331-1348 (2001)

[13] Liu, X., Sun, R., Kang, S.B., Shum, H.Y.: Directional Histogram Model for ThreeDimensional Shape Similarity. In: CVPR 2003, vol. 1, pp. 813-820 (2003)

[14] Adán, A., Cerrada, C., Feliú, V.: Modeling Wave Set: Definition and Application of a new Topological Organization for 3D Object Modeling. Computer Vision and Image Understanding 79, 281-307 (2000)

[15] Adán, A., Adán, M.: A Flexible Similarity Measure for 3D Shapes Recognition. IEEE Transactions on Pattern Analysis and Machine Intelligence 26(11), 1507-1520 (2004) 